\title{
Comparison of a single-use, digital flexible ureteroscope with a reusable, fiberoptic ureteroscope for management of patients with urolithiasis
}

\author{
Panagiotis Mourmouris ${ }^{1}$, Lazaros Tzelves ${ }^{1}$, Grigorios Raptidis ${ }^{2}$, Marinos Berdempes ${ }^{1}$, \\ Titos Markopoulos ${ }^{1}$, Grigorios Dellis ${ }^{2}$, Ioannis Siafakas ${ }^{2}$, Andreas Skolarikos ${ }^{1}$ \\ ${ }^{1}$ National and Kapodistrian University of Athens, $2^{\text {nd }}$ University Department of Urology, Sismanoglio Hospital, Athens, Greece; \\ 2251 Airforce General Hospital, Urology Department, Athens, Greece.
}

\begin{abstract}
Summary Objectives: Ureteroscopy is one of the commonest procedures performed to manage urolithiasis. Flexible ureteroscopy has been traditionally based on reusable, fiber-optic ureteroscopes. Technology advancements permitted the development of single-use scopes with digital image. The aim of this study is to compare efficacy and safety between a reusable, fiberoptic ureteroscope with a single-use, digital scope.

Patients and methods: We collected data based on chart review from a prospectively collected database on a tertiary, high-volume hospital in Greece. Baseline, perioperative and postoperative data were gathered and analyzed. Chi-square and Fisher's exact test was used to compare qualitative data and unpaired t-test for continuous data, with a statistical significance set at $a=0.05$.

Results: 40 patients underwent flexible ureteroscopy with a single-use digital scope, while 37 with the reusable scope. The two groups were matched regarding baseline characteristics and stone-related parameters. After data analysis, a shorter operative time in favor of single-use flexible ureteroscope was detected (45 vs $65 \mathrm{~min}, p=0.001$ ), while safety was also in favor of this type of scope with a significantly higher immediate stonefree rate ( $70 \%$ vs $43 \%, p=0.005)$. Overall complications did not differ between the two groups, although a lower sepsis rate was detected in patients treated with single-use scope.

Conclusions: Our findings indicate that single-use, digital ureteroscopes are a viable alternative for flexible ureteroscopy and management of urolithiasis, especially in centers with deficient facilities for sterilization and ensured funds for more expensive reusable scopes.
\end{abstract}

KEY WORDS: Urolithiasis; Flexible ureteroscope; Kidney stone disease; Digital ureteroscope; Single-use ureteroscope; Fiber-optic ureteroscope; Reusable ureteroscope.

Submitted 11 July 2021; Accepted 25 July 2021

\section{INTRODUCTION}

Geography and climatic changes along several regions affect kidney stone disease prevalence. In Greece, urolithiasis is found to affect around 15\% of population (1), while in contrast US citizens suffer from kidney stones to a lesser extent of around 9\% (2). The total annual cost directed to this condition reaches around 5.3 billion $\$$ in
US $(3,4)$. Technological advances have contributed to the development of new technologies and techniques, that play a major role in the management of this disease. Semirigid and flexible ureteroscopes constitute a basic component of endourologist armamentarium.

After the first appearance of digital technology in ureteroscopes in 2004 (5) many more followed. Reusable instruments offer repeatability but with uncertain endurance. Legemate et al. (6) report that shaft bending, kinking and dent of coating is an issue that appears not seldom in everyday use. Moreover sterilization procedures that apply for reusable instruments are accompanied by increased costs, necessary time intervals between operations and trained staff members (7). The first single-use digital flexible ureteroscope, was launched back in 2015 (8). Equipped with a tip diameter of 7.7 Fr, outer diameter of 9.5 Fr, a wide enough working channel for baskets and laser fibers (3.6 Fr), digital imaging and deflection angle up to 270 degrees, it is a great aid for the endourologist 8. A wide variety of movements, such as pronation/ supination, downward and upward deflection, along with back and forth movements, offers a great degree of freedom for the operator (8).

Literature search revealed data of in vitro/in vivo studies (8) and cadavers (9), while initial results on comparison with reusable fiberoptic ureteroscope showed better results in the single-use group regarding procedural time, failure of procedure and complications. Despite the convincing results more studies should be conducted to reach safe conclusions. The aim of this study is to compare intra- and postoperative complications and parameters while using a single-use, digital flexible ureteroscope, in comparison with a reusable fiberoptic ureteroscope in a prospective cohort of matched population for their baseline characteristics and their disease.

\section{Patients ANd methods}

Study design

Data collection was performed from an ongoing prospective database regarding patients treated for urolithiasis with a single-use, digital or reusable fiberoptic ureteroscope. 
Chart review for patient demographic characteristics, stone disease parameters and perioperative details was performed. Cross-match of patients for confounders was also performed (stone disease parameters, age, ASA score).

\section{Settings}

Data were derived from Second Urology Department in Sismanoglio, a tertiary Hospital in Greece, which is considered a reference center for stone disease, between a 12 month period (06/2017-06/2018). A high volume of fURS cases is performed yearly at out center (> 100).

\section{Participants}

Inclusion criteria were: patients older than 18 y/o with diagnosed stone disease based on imaging studies (ultrasound, CT scan or x-Ray). Patients with non-radiopaque stone disease, history of urinary tract neoplasm or those undergoing a diagnostic workup for hematuria were excluded. The Ethics Committee of Hospital approved study protocol and patients were informed about inclusion and signed informed consent in case of participation. The study was conducted according to the principles of Helsinki Declaration. Assignment to the specific treatment arm was done according to patient choice after being informed for the potential choices and availability of equipment.

\section{Variables}

The demographic profile of patients was based on collection of data like age, gender, American Society of Anesthesiologists (ASA) score according to anesthesiologic evaluation, side of disease, presence of bacteriuria and potential anatomic malformation of kidneys.

Perioperative variables like operation room time, technique and equipment used during ureteroscopy, complications and duration of hospital stay, stone free rates, as well as stone disease characteristics were also gathered (stone location, maximum diameter, and total burden).

\section{Data sources and measurements}

In the study six experienced urologists performed the total number of cases either with LithoVue ${ }^{T M}$ (Boston Scientific) single-use, digital ureteroscope or the Flex X2 (Karl Storz) fiberoptic, reusable fiberoptic ureteroscope. The exact same equipment was used between the two groups concerning laser fiber, baskets and other retrieval devices, as well as access sheaths in order to minimize confounding effect. Antibiotic prophylaxis was administered to all patients according to existing EAU Guidelines and preoperative urine culture results.

Data regarding perioperative details were recorded by the urologist, the residents who were present in the operating room or by scrub nurses. The total operative time was defined as the length of time from ureteroscope entry until completion of stone pulverization. The patient stone burden was determined based on the most accurate imaging modality, while in order to categorize a patient stone free, no fragments of residual stone disease or clinically insignificant fragments $<2 \mathrm{~mm}$ (CIFRs) should have been identified during patient follow-up with imaging tests.

\section{Bias}

In order to limit confounding bias, we performed a cross- matching of cases regarding baseline characteristics and stone disease parameters.

\section{Statistical methods}

Categorical variables are described as proportions, while Fisher's exact test or chi-square test were used for comparing them. Continuous data are presented as mean \pm standard deviation or medians and analyzed using unpaired Student's t-test or Mann-Whitney U test. The choice of Mann-Whitney or t-test according to normal distribution was determined based on assessment of Q-Q plots and Shapiro-Wilk test. Statistical significance was set at $\mathrm{a}=0.05$. All analyses were done with IBM SPSS Statistics 25.0 software (SPSS Inc., Chicago, IL.).

\section{RESULTS}

During the study recruitment period, 77 patients were treated, including 40 in the single-use scope group and the rest 37 in reusable scope group. The two groups were balanced with respect to mean age $(55.73 \pm 13.47$ vs 55 $\pm 11.2, p=0.797)$, use of access sheath $(88 \%$ vs $92 \%$, $\mathrm{p}=0.713)$ and semirigid ureteroscope (60\% vs $59 \%$, $\mathrm{p}>0.99$ ) but more men (55\% vs 38\%) and more patients with positive urine culture preoperatively ( $23 \%$ vs $11 \%$ ) were included in the single-use scope group, although this did not reach statistical significance (Table 1).

Maximum stone size didn't differ between the groups (12.63 vs $12.52 \mathrm{~mm}, \mathrm{p}=0.914$ ), while the most common stone location was renal pelvis and lower pole calyces. CT scan was used more frequently for diagnosis in the singleuse scope group $(78 \%$ vs $57 \%, p=0.087)$ and hydronephrosis was more frequent, but this didn't reach statistical significance (Table 2).

Regarding the laser fiber used from stone fragmentation, in single-use scope group the $270 \mu \mathrm{m}$ was more frequently used $(57.5 \%$ vs 30\%, p $=0.092)$ but results didn't differ significantly. All patients received post-operative insertion of a double-J stent and a similar proportion in both groups was pre-stented. Ancillary use of basket for stone removal and laser setting use for stone fragmentation were also similar between the two groups (Table 3). Median operative time (45 vs $65 \mathrm{~min}, \mathrm{p}<0.001$ ), sepsis rate $(0 \%$ vs $11 \%, p=0.049)$ and stone free rate at day one after surgery $(78 \%$ vs $43 \%, \mathrm{p}<0.001)$ favored use of single-use scope. No intraoperative complications were observed in both groups. Finally, length of hospital stay and rates of

\section{Table 1.}

Baseline demographic characteristics of patients.

\begin{tabular}{|l|c|c|c|}
\hline Characteristic & $\begin{array}{c}\text { Single-use, } \\
\text { digital }(\mathbf{n}=\mathbf{4 0})\end{array}$ & $\begin{array}{c}\text { Reusable, fiber-optic } \\
\text { ureteroscope (n= 37) }\end{array}$ & P-value \\
\hline Mean age \pm SD & $55.73 \pm 13.47$ & $55 \pm 11.2$ & 0.797 \\
\hline Male sex - no. (\%) & $22(55)$ & $14(38)$ & 0.172 \\
\hline ASA Score $\leq 2$ - no. (\%) & $39(98)$ & $34(22)$ & 0.441 \\
\hline Positive urine culture - no. (\%) & $9(23)$ & $4(11)$ & 0.228 \\
\hline Kidney laterality left - no. (\%) & $19(47)$ & $17(46)$ & $>0.999$ \\
\hline Present renal anomaly - no. (\%) & $4(10)$ & $1(3)$ & 0.359 \\
\hline Use of semirigid ureteroscope - no. (\%) & $24(60)$ & $22(59)$ & $>0.999$ \\
\hline Use of access sheath - no. (\%) & $35(88)$ & $34(92)$ & 0.713 \\
\hline
\end{tabular}


Table 2.

Stone characteristics.

\begin{tabular}{|l|c|c|c|}
\hline Parameter & $\begin{array}{c}\text { Single-use, } \\
\text { digital }(\mathbf{n}=\mathbf{4 0})\end{array}$ & $\begin{array}{c}\text { Reusable, fiber-optic } \\
\text { ureteroscope }(\mathbf{n}=\mathbf{3 7})\end{array}$ & P-value \\
\hline Median number of stones (mm) & 1.07 & 1.65 & 0.625 \\
\hline Median maximum stone diameter (mm) & 12.63 & 12.52 & 0.914 \\
\hline Median total stone burden (mm) & 17.36 & 15.22 & 0.284 \\
\hline Present pre-operative hydronephrosis - no. (\%) & $20(50)$ & $15(41)$ & 0.494 \\
\hline Use of CT scan for diagnosis - no. (\%) & $31(78)$ & $21(57)$ & 0.087 \\
\hline Pelvicalyceal location of stones - no. (\%) & $2(5)$ & $7(19)$ & 0.698 \\
Upper ureter & $16(40)$ & $10(27)$ & \\
Renal pelvis & $2(5)$ & $2(5)$ & \\
Middle renal pole & $5(12.5)$ & $4(11)$ & \\
Lower renal pole & $1(2.5)$ & $1(3)$ & \\
Renal pelvis/Upper pole & $2(5)$ & $11(30)$ & \\
Renal pelvi/Middle pole & $10(25)$ & $0(0)$ & \\
Renal pelvis/Lower pole & $1(2.5)$ & $1(3)$ & \\
Multiple calyces & & & \\
\hline
\end{tabular}

Table 3.

Procedural characteristics.

\begin{tabular}{|c|c|c|c|}
\hline Characteristic & $\begin{array}{l}\text { Single-use, } \\
\text { digital }(n=40)\end{array}$ & $\begin{array}{l}\text { Reusable, fiber-optic } \\
\text { ureteroscope }(n=37)\end{array}$ & P-value \\
\hline $\begin{array}{l}\text { Use of basket for remaining stone } \\
\text { fragments - no. }(\%)\end{array}$ & $12(30)$ & $13(35)$ & 0.902 \\
\hline Pre-operative JJ stent - no. (\%) & $14(35)$ & $13(35)$ & $>0.999$ \\
\hline Post-operative JJ stent - no. (\%) & $40(100)$ & $37(100)$ & \\
\hline $\begin{array}{l}\text { Size of laser fiber used for stone } \\
\text { fragmentation - no. }(\%)\end{array}$ & & & 0.092 \\
\hline $270 \mu \mathrm{m}$ & $23(57.5)$ & $11(30)$ & \\
\hline $365 \mu \mathrm{m}$ & $9(22.5)$ & $15(41)$ & \\
\hline $270 \& 365 \mu \mathrm{m}$ & $6(15)$ & $8(20)$ & \\
\hline Laser settings used - no. (\%) & & & 0.092 \\
\hline Dusting & $25(62.5)$ & $14(38)$ & \\
\hline Chipping & $2(5)$ & $2(5)$ & \\
\hline Dusting \& Popcorn & $6(15)$ & $9(24)$ & \\
\hline Chipping \& Popcorn & $5(12.5)$ & $10(27)$ & \\
\hline
\end{tabular}

Table 4.

Intraoperative and postoperative outcomes.

\begin{tabular}{|l|c|c|c|}
\hline Outcome & $\begin{array}{c}\text { Single-use, } \\
\text { digital }(\mathbf{n}=\mathbf{4 0})\end{array}$ & $\begin{array}{c}\text { Reusable, fiber-optic } \\
\text { ureteroscope }(\mathbf{n}=\mathbf{3 7})\end{array}$ & P-value \\
\hline Median operative time (min) & 45.00 & 65.00 & $<0.001$ \\
\hline Mean length of stay in hospital \pm SD (days) & $1.75(1.96)$ & $1.38(0.64)$ & 0.261 \\
\hline Immediate stone free status - no. (\%) & $28(70)$ & $16(43)$ & $<0.005$ \\
\hline $\begin{array}{l}\text { Stone free status } 24 \text { hours postoperatively } \\
\text { - no. (\%) }\end{array}$ & $31(78)$ & $16(43)$ & $<0.001$ \\
\hline Intraoperative complications - no. (\%) & $0(0)$ & $0(0)$ & \\
\hline Postoperative complications - no. (\%) & $2(5)$ & $6(16)$ & 0.144 \\
\hline Postoperative fever - no. (\%) & $2(5)$ & $6(16)$ & 0.144 \\
\hline Postoperative hematuria - no. (\%) & $2(5)$ & $3(8)$ & 0.667 \\
\hline Postoperative sepsis - no. (\%) & $0(0)$ & $4(11)$ & 0.049 \\
\hline
\end{tabular}

post-operative fever and macroscopic hematuria didn't differ significantly between the two groups (Table 4).

\section{Discussion}

Innovations in equipment technology resulted in improvement of clinical outcomes and rendered endo- scopic management of stone disease the gold-standard (10). In this study we performed a comparison of clinical outcomes with the use of a disposable, single-use and digital flexible ureteroscope or a re-usable, fiber-optic flexible ureteroscope. The single-use ureteroscope performance in animal studies showed promising results regarding its efficacy and safety. The risk of transmitting potentially fatal infections with repeated use of duodenoscopes (11) or ureteroscopes (12), along with the associated costs for sterilization and maintenance, favor the adoption of single-use scopes, when efficacy is similar. A mean maximum deflection of 270 degrees when used with an empty working channel was found by Winship (13), while after insertion of a laser fiber, an 8.3 degrees reduction in deflection angle was noted. Duration of this type of scope proved satisfying since after 200 full deflections, a mean 21.8 degrees loss was detected, thus maintaining the desired deflection of $>250$ degrees (13). This bench-top study also indicates no distortion of digital imaging after laser insertion through the working channel (13).

In our study, we found a significant reduction in operative time equal to 20 minutes, although the two groups were similar in term of demographic characteristics, stone size and location. The reduction of operative time by 30\% can save time for completion of further cases, reduce the physical burden of staff and the total associated costs. The lower weight of the single-use scope, which adds dexterity to the operator and reduces physical strain during ureteroscopy can be a possible explanation for these findings (6). Endourologists frequently suffer from orthopedic problems in wrists/upper arms, as reported by Healy et al. (14) in their survey, where $32 \%$ of urologists performing fURS responded dealing with such issues. Proietty et al. (15) reported that such a single-use scope is 10-300\% lighter with camera head and light cable attached when compared to its counterpart reusable digital/fiberoptic scopes. Less physical strain of endourologist might contribute to reduced operating time detected to our study. Improved visual field during use of digital imaging also contributes to reduction of operative time and increases safety intraoperatively, according to Somani et al. (16). Another important advantage offered by the single use nature is that inexperienced users like residents, can handle it without the excess fear of scope breakage. According to Mager et al. (17) and Kam et al. (18), low-volume centers (< 51-60 fURS yearly) might benefit from establishing a single-use scope based program, while high-volume centers (> 10/month) are more likely to save costs with reusable scopes, especially when they are handled properly. Of course, current local market prices in each country may dictate different adaptations in a case-specific scenario. To ensure the superiority of a single-use scope regarding cost-effectiveness, further dedicated studies are needed.

Results of the CROES Global study (19) imply an $80 \%$ stone-free rate (SFR) for stones $<15 \mathrm{~mm}$ after a single fURS, which is comparable with the $78 \%$ SFR found in this study sample when using a single-use scope, for a median stone size of $12.63 \mathrm{~mm}$. Of course, the several definitions used for stone-free rate across studies, may weaken these results, but the increased SFR seems promising, since this is the main primary outcome and the main determining factor to guide future management of 
patients with urolithiasis. The fact that this a relatively new equipment and learning curve did not negatively impact perioperative results, further strengthens its use. Post-operative fever shows a reported incidence equal to $0-10.8 \%$ (20-23) after operating in the urinary tract for stones less than $20 \mathrm{~mm}$, using fURS. The main contributing factors are female gender, increased body mass index, positive pre-operative urine culture, increased operative time and increased renal pelvic pressure. In our study we detected a rate of $5 \%$ in single-use scope group, which lies in agreement with existing literature. The reduction in operative time can be a protective factor for post-operative fever and sepsis when using single-use scopes, mainly due to less extend increase of renal pelvic pressure. Patients in single-use scope group also suffered less hematuria and sepsis, which is quite important considering morbidity and mortality of urosepsis. This study has certain limitations. Since this is not a randomized controlled trial, there is the possibility for selection bias, which we tried to minimize by cross-matching the groups for baseline demographic characteristics and stone disease parameters. The learning curve and the limited follow-up could also obscure the results regarding stone-free rates.

\section{Conclusions}

This study compares a single-use, digital flexible ureteroscope with a re-usable, fiber-optic flexible ureteroscope for treatment of stone disease. The fact that single-use scope significantly decreases procedural duration and sepsis rates, while increased immediate-stone free rates, makes it a viable option for management of stone disease. Further randomized trials and cost-effectiveness studies are needed to confirm these results.

\section{REFERENCES}

1. Stamatiou K, Karanasiou VI, Lacroix R et al. Prevalence of urolithiasis in rural Thebes, Greece. Rural Remote Health. 2006; 6:610.

2. Scales CD J, Smith AC, Hanley JM, Saigal CS. Prevalence of kidney stones in the United States. Eur Urol. 2012; 62:160-165.

3. Ghani KR, Sammon JD, Karakiewicz PI, et al. Trends in surgery for upper urinary tract calculi in the USA using the Nationwide Inpatient Sample: 1999-2009. BJU International. 2013; 112:224-230.

4. Saigal CS JG, Timilsina AR. Direct and indirect costs of nephrolithiasis in an employed population: opportunity for disease management? Kidney Int. 2005; 68:1808-1814.

5. Shah K MM, Knudsen B. Prospective randomized trial comparing 2 flexible digital ureteroscopes: ACMI/Olympus Invisio DUR-D and Olympus URF-V. Urology. 2015; 85:1267-1271.

6. Legemate JD, Kamphuis, G. M., Freund, J. E., et al. Durability of flexible ureteroscopes: a prospective evaluation of longevity, the factors that affect it, and damage mechanisms. Eur Urol Focus. 2019; 5:1105-1111.

7. Semins MJ GS, Allaf ME, Matlaga BR. Ureteroscope cleaning and sterilization by the urology operating room team: the effect on repair costs. J Endourol. 2009; 23:903-905.

8. Dale J, Kaplan AG, Radvak D, Shin R, et al. Evaluation of a novel single-use flexible ureteroscope. J Endourol. 2021; 35:903-907.

9. Proietti S DL, Molina W, Doizi S, et al. Comparison of new sin- gle-use digital flexible ureteroscope versus nondisposable fiber optic and digital ureteroscope in a cadaveric model. J Endourol. 2016; 30:655-659.

10. Kartal I, Baylan B, Cakici MC, et al. Comparison of semirigid ureteroscopy, flexible ureteroscopy, and shock wave lithotripsy for initial treatment of 11-20 $\mathrm{mm}$ proximal ureteral stones. Arch Ital Urol Androl. 2020; 92:39-44.

11. Epstein L, Hunter JC, Arwady MA, et al. New Delhi metallo-囚lactamase-producing carbapenem-resistant Escherichia coli associated with exposure to duodenoscopes. JAMA. 2014; 312:1447-1455.

12. Chang CL SL, Lu CM, et al. Outbreak of ertapenem-resistant Enterobacter cloacae urinary tract infections due to a contaminated ureteroscope. J Hosp Infect. 2013; 85:118-24.

13. Winship B WD, Carlos E, Li J, et al. Avoiding a lemon: performance consistency of single-use ureteroscopes. J Endourol. 2019; 33:127-131.

14. Healy KA PR, Cleary RC, Colon-Herdman A, Bagley DH. Hand problems among endourologists. J Endourol. 2011; 25:1915-1920.

15. Proietti S, Somani B, Sofer, et al. The "Body Mass Index" of flexible ureteroscopes. J Endourol. 2017; 31:1090-1095.

16. Somani BK, Al-Qahtani SM, de Medina SD, Traxer O. Outcomes of flexible ureterorenoscopy and laser fragmentation for renal stones: comparison between digital and conventional ureteroscope. Urology. 2013; 82:1017-1019.

17. Mager R KM, Höfner T, Frees S, et al. Clinical outcomes and costs of reusable and single-use flexible ureterorenoscopes: a prospective cohort study. Urolithiasis. 2018; 46:587-593.

18. Kam J, Yuminaga Y, Beattie K, et al. Single use versus reusable digital flexible ureteroscopes: A prospective comparative study. Int J Urol. 2019; 26: 999-1005.

19. Skolarikos A, Gross AJ, Krebs A, et al. Outcomes of flexible ureterorenoscopy for solitary renal stones in the CROES URS Global Study. J Urol. 2015; 194:137-143.

20. Kourambas J DF, Munver R, Preminger GM. Nitinol stone retrieval-assisted ureteroscopic management of lower pole renal calculi. Urology. 2000; 56:935-939.

21. Hollenbeck BK ST, Faerber GJ, Wolf JS. Flexible ureteroscopy in conjunction with in situ lithotripsy for lower pole calculi. Urology. 2001; 58:859-863.

22. Jung H NB, Osther PJ. Retrograde intrarenal stone surgery for extracorporeal shock-wave lithotripsy-resistant kidney stones. Scand J Urol Nephrol. 2006; 40:380-384.

23. El-Nahas AR IH, Youssef RF, Sheir KZ. Flexible ureterorenoscopy versus extracorporeal shock wave lithotripsy for treatment of lower pole stones of 10-20 $\mathrm{mm}$. BJU International. 2012; 110:898-902.

\section{Correspondence}

Panagiotis Mourmouris, MD - thodoros13@yahoo.com

Lazaros Tzelves, MD, Msc (Corresponding Author) - lazarostzelves@gmail.com Andreas Skolarikos, MD - andskol@yahoo.com

$2^{\text {nd }}$ University Department of Urology, Sismanoglio Hospital, Sismanogliou 37, Athens (Greece)

Grigorios Raptidis, MD - gregrapt@otenet.gr

Marinos Berdempes, MD - marinosberdebes@hotmail.com

Titos Markopoulos, MD - titosmark@gmail.com

Grigorios Dellis, MD - grdellis@yahoo.gr

Ioannis Siafakas, MD - sfksgnns@yahoo.com

251 Airforce General Hospital, Urology Department, Athens (Greece) 